\title{
Rapid Determination of Ascorbic Acid in Fresh Vegetables and Fruits with Electrochemically Treated Screen-Printed Carbon Electrodes
}

\author{
Ling Xiang ${ }^{1,2}$, Hua Ping ${ }^{1,2}$, Liu Zhao ${ }^{1,2}$, Zhihong Ma ${ }^{1,2}$, and Ligang Pan ${ }^{1,2}$ \\ ${ }^{1}$ Beijing Research Center for Agrifood Testing and Farmland Monitoring, \\ Beijing 100097, P. R. China \\ ${ }^{2}$ Beijing Research Center for Information Technology in Agriculture, \\ Beijing 100097, P. R. China \\ lgpan@nercita.org.cn
}

\begin{abstract}
This study demonstrates a new electrochemical method for rapid determination of ascorbic acid in real samples with electrochemically pretreated screen-printed carbon electrodes. Cyclic voltammetry results indicated that the electrochemically activated electrodes possessed an excellent electrocatalytic activity toward ascorbic acid oxidation and can be successfully used for its selective and sensitive determination in fresh vegetables and fruits. The proposed method was further validated by means of specificity assay with ascorbate oxidase and a comparison with an accurate method such as high-performance liquid chromatography using ultraviolet detection. The electrodes presented the advantages over other existing methods such as mass production and without artificial modification. This study essentially offers a facile and reliable method for rapid determination of ascorbic acid in food analysis with high selectivity and sensitivity as well as low cost.
\end{abstract}

Keywords: Ascorbic Acid, Electrochemical Pretreatment, Screen-Printed Electrodes, Vegetables, Fruits.

\section{Introduction}

Increasing evidences have revealed that ascorbic acid (AA) possesses lots of different functions in the physiological and pathological processes, such as an important antioxidant and free radical scavenger in neuroprotection, an essential cofactor for biosynthesis of neuropeptides, and an effective neuromodulator of dopamine- and glutamate-mediated neurotransmission [1]. As fresh vegetables and fruits are the most important suppliers of AA in normal diets, the determination of AA can offer a marker for food analysis and quality evaluation.

There are several reported methods for the determination of AA in foodstuffs, including chromatography [2], spectrophotometry [3], and titration [4], etc.. However, these methods usually require complex steps, which may increase the chance of AA oxidation during the pretreatment of fresh samples. On the other hand, some methods may lack good specificity and require high-cost equipments. Thus, the development 
of rapid, facile, and reliable methods for AA determination is particularly important in food industry. Recently, electrochemical methods have shown unique advantages in rapid determination of AA [5]. AA can be electrochemically oxidized through a two electron and one-proton pathway followed by an irreversible hydrolysis process to produce an electroinactive product at neutral $\mathrm{pH}$ [6]. Such an electrochemical property of AA can be used to realize direct electrochemical determination of AA with simple steps. But the high over-potential of AA oxidation at the conventional electrodes leads to poor specificity in real samples which also contain other electroactive substances, such as thiols and phenols [7]. Focused on the solution of this problem, most research used chemically modified electrodes [8] or the additional use of ascorbate oxidase (AAox) to differentiate the current response for AA from the total response obtained [9]. O'Connell et al. proposed a sensor modified with electropolymerized aniline (PANI) to selectively catalyze the oxidation of AA at low potential $(+100 \mathrm{mV})$ so as to minimize the effects of most common interfering agents in juices and pharmaceuticals [10]. A carbon paste electrode modified with $\mathrm{SiO}_{2} / \mathrm{SnO}_{2} /$ phosphate/Meldola's blue was used to study the electrocatalytic oxidation of AA in commercial fruit juices [11]. Civit et al. successfully demonstrated amperometric determination of AA in several kinds of fruits and vegetables using a disposable screen-printed electrode modified with electrografted o-aminophenol film [12]. With the appearance of carbon nanotubes (CNTs), recent electrochemical studies have revealed that CNTs can facilitate the oxidation of AA at a low potential, and demonstrated possible application of CNTs for selective determination of AA [13]. Crevillén et al. developed carbon nanotube-modofied disposable detectors in microchip capillary electrophoresis for AA determination in pharmaceutical quality control [14]. However, Most of the above mentioned mehods showed a certain degree of complexity in the phase of electrode modification.

This study exploits a new electrochemical sensor for determination of AA in fresh vegetables and fruits with an electrochemical pretreated disposable screen printed carbon electrode. The integrated screen printed carbon electrode, which consists of a carbon counter electrode, a silver pseudo-reference electrode and a carbon working electrode, is amenable to rapid fabrication and mass production. In addition, this method demonstrated here just requires simple activation of electrodes to enhance the selectivity of AA determination without artificial modification, and could find some interesting applications in food and pharmaceutical industry.

\section{Experimental Methods}

\subsection{Reagents and Solutions}

All chemicals were of analytical grade or higher and used as received. Aqueous solu-

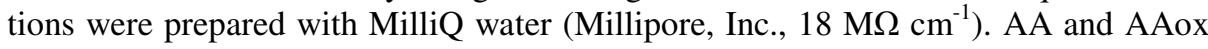
(Cucurbita species, EC 1.10.3.3) were all purchased from Sigma and used as supplied. A stock solution of AA (1.0 mM) was prepared in $0.1 \mathrm{M}$ phosphate buffer at $\mathrm{pH} 7.0$ just before use. Oxalic acid and other reagents were all purchased from Beijing Chemical Co., Ltd. (Beijing, China). AAox was used to prove the absence of collateral reactions by means of specificity assays. The enzyme (250 U) was dissolved in 
$1.0 \mathrm{~mL} 0.1 \mathrm{M}$ phosphate buffer ( $\mathrm{pH} 7.0$ ), and divided into portions and stored at -40 ${ }^{\circ} \mathrm{C}$ until use. One portion was taken out and diluted with $0.1 \mathrm{M}$ phosphate buffer $(\mathrm{pH}$ 7.0) to get a fresh AAox solution with the concentration of $25.0 \mathrm{U} / \mathrm{mL}$ for use.

\subsection{Electrode Preparation}

The planar screen printed carbon electrodes (SPCEs) are designed by our lab and fabricated by Beijing Meiborui Co., Ltd. (Beijing, China) as previously described. ${ }^{15}$ The electrode $4.0 \mathrm{~cm} \times 1.2 \mathrm{~cm} \times 0.05 \mathrm{~cm}$ (length $\times$ width $\times$ height) consists of three main parts which are a carbon counter electrode, a silver pseudo-reference electrode and a carbon working electrode. The carbon working surface is $4 \mathrm{~mm}$ in diameter. The $\mathrm{Ag} / \mathrm{AgCl}$ reference electrode was formed by applying $0.3 \mathrm{M} \mathrm{FeCl}_{3}$ solution on the silver electrode for $10 \mathrm{~min}$ [15].

The fabricated SPCEs were first sequentially sonicated in ethanol, $3.0 \mathrm{M} \mathrm{HNO}_{3}$, 1.0 $\mathrm{M} \mathrm{KOH}$, and distilled water each for $3 \mathrm{~min}$., and then typical electrochemical pretreatment of SPCEs was carried out by applying $+2.5 \mathrm{~V}$ for $60 \mathrm{~s}$ in $1.0 \mathrm{M} \mathrm{NaOH}$ ( $v s . \mathrm{Ag} / \mathrm{AgCl}$ reference electrode on the same SPCE).

\subsection{Sample Preparation}

Fresh orange and cabbage samples were obtained from a local supermarket. The operative processes were the usual ones: green cabbage leaves were washed in running water, drained, and finally weighed (about $50 \mathrm{~g} / \mathrm{sample}$ ). Oranges were peeled and cut into suitable, representative portions of the proper weights (about $50 \mathrm{~g} / \mathrm{sample}$ ).

Each sample was prepared by weighing a sufficient amount of vegetable or fruit to provide ca. $10 \mathrm{mg}$ of AA/sample. $125 \mathrm{~mL} 0.1 \%(\mathrm{w} / \mathrm{w})$ oxalic acid was immediately added to each sample, and the sample was homogenized with a Braun knife homogenizer for a few seconds. The homogenate was immediately submitted to $5000 \mathrm{rpm}$ in a refrigerated centrifuge for $5 \mathrm{~min}$. An aliquot of the clear supernatant solution was filtered through a $0.45 \mu \mathrm{m}$ pore-size filter (Millipore, Bedford, MA) before being injected into the system of high-performance liquid chromatography (HPLC).

\subsection{Electrochemical Measurements}

Electrochemical measurements were carried out on a computer-controlled electrochemical analyzer (CHI660C, CHI, TX), and were performed by placing a drop of the corresponding solution on the working area. A $0.10 \mathrm{M}$ phosphate buffer solution ( $\mathrm{pH}$ 7.0) was used as supporting electrolyte for the electrochemical studies. For real sample analysis, $1.0 \mathrm{~mL}$ of the clear supernatant solution containing AA after centrifugation was first mixed with $1.0 \mathrm{~mL}$ of the working buffer solution $(0.1 \mathrm{M}$ phosphate buffer at $\mathrm{pH}$ 7.0), and then a $50 \mu \mathrm{L}$ drop of the corresponding solution was placed on the working area before measurement.

\subsection{HPLC Analysis}

Determination of AA by HPLC was performed in a Waters 2690 HPLC system (Milford, Massachusetts), with a column $(5 \mu \mathrm{m} ; \mathrm{C} 18 ; 150 \times 3.9 \mathrm{~mm})$. Detection was 
done by ultraviolet (UV) absorption at $254 \mathrm{~nm}$ (Waters 2487 Tunable Absorbance Detector) [16]. At a flow rate of $0.6 \mathrm{~mL} / \mathrm{min}$ (Waters 2690 Pump), the retention time for AA was 2.75 min. Identification of the AA peak was carried out using a highly purified standard solution prepared as $20 \mathrm{ppm}$ in $0.1 \%$ (w/w) oxalic acid.

The HPLC mobile phase was a $97 \%: 3 \% \mathrm{~V} / \mathrm{V}$ mixture of oxalic acid $(0.1 \%(\mathrm{w} / \mathrm{w})$, adjusted to $\mathrm{pH} 2.5$ before mixing) and methanol, which was filtered through a 0.45 $\mu \mathrm{m}$ pore-size filter (Millipore, Bedford, MA).

\subsection{Specificity Assays}

Aliquots of $5.0 \mu \mathrm{L}$ of the enzyme solution $(25.0 \mathrm{U} / \mathrm{mL})$ were added into the $1.0 \mathrm{~mL}$ mixture, which was composed of clear supernatant solution obtained in the sample preparation and 0.1 M phosphate buffer at $\mathrm{pH} 7.0$ (v/v 1:1), in order to achieve utter oxidation of the AA present in a period not longer than $15 \mathrm{~min}$.

\section{Results and Discussion}

\subsection{AA Oxidation at the Fresh and Pretreated SPCEs}

As shown in Fig. 1, at a fresh SPCE without activation, the oxidation of AA began irreversibly at $0 \mathrm{~V}$, showing poor electrochemical kinetics with high over-potential and low electron transfer rate. McCreery et al. have systematically studied electrochemical reactions at carbon electrodes and demonstrated that the oxidation of AA is an inner-sphere reaction with electron-transfer kinetics sensitive to the electrode surface [17]. Thus the electrode activity shows a dramatic effect on electrochemical oxidation property of AA, which is generally determined by one or more of the following factors: (1) surface microstructure, (2) surface cleanliness, (3) hydrophilicity/hydrophobicity, (4) electronic structure, and (5) surface functional groups.

It is well known that carbon electrodes are often necessarily preconditioned by electrochemical pretreatment to enhance the electron transfer properties at the electrode surface [18]. No matter electrochemical pretreatment are carried out in an acid, base or neutral solution, the main purpose of pretreatment on SPCEs is to remove organic ink constitutes or contaminates and to increase surface roughness or the density of oxygenated groups [19]. To find appropriate electrochemical pretreatment for optimum electrochemical performances of SPCEs designed by our group, different electrolytes and conditions were studied using potassium ferricyanide as an electrochemical probe. By gradually changing the concentration of $\mathrm{NaOH}$, applied potential as well as pretreatment time, an electrochemical activation procedure, applying $+2.5 \mathrm{~V}$ for $60 \mathrm{~s}$ in $1.0 \mathrm{M} \mathrm{NaOH}$ aqueous solution, was finally selected as the most reproducible and advantageous. As shown in Fig. 2(A), the response of a fresh SPCE without electrochemical activation was poor with a large peak separation of oxidation and reduction $(\triangle \mathrm{Ep}>1000 \mathrm{mV})$, indicating a slow electron transfer rate and a irreversible electrochemical process. As to the electrode after pretreated in $1.0 \mathrm{M} \mathrm{NaOH}$ at $+2.5 \mathrm{~V}$ for $60 \mathrm{~s}, \triangle \mathrm{Ep}$ was reduced to ca. $160 \mathrm{mV}$, showing a rapid electron transfer rate and a quasi-reversible electrochemical response. The result also showed that the current response of $\mathrm{Fe}(\mathrm{CN})_{6}{ }^{3-} /^{4-}$ at pretreated SPCE 
obviously increased, compared with the fresh SPCE, due to the improvement of electron transfer rate and electrode surface roughness caused by the electrochemical activation procedure.

Unlike the fresh SPCE just after rinse in different solutions, AA oxidation started at $-0.4 \mathrm{~V}$ after electrode pretreatment shown in Fig. 2(B), which was obviously more negative compared with that at the fresh electrode. This result clearly indicated that the effective electrochemical activation leaded to a faster electron-transfer kinetics for the AA oxidation, which benefited for selective determination of AA at lower potential on the pretreated electrode. The comparison of the voltammograms obtained for AA oxidation at the SPCEs before and after pretreatment also showed the enhancement of current response of AA and background charging current, due to the increase of electrode surface caused by electrochemical pretreatment.

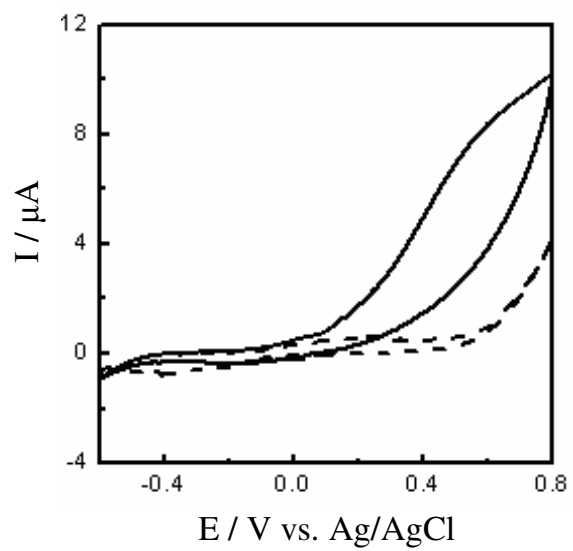

Fig. 1. CVs obtained at a fresh SPCE in $0.1 \mathrm{M}$ phosphate buffer (pH 7.0) in the absence (dashed line) and presence (solid line) of $0.50 \mathrm{mM}$ AA before electrochemical pretreatment. Scan rate, $100 \mathrm{mV} \mathrm{s}^{-1}$.

In addition, the electrochemical activated SPCEs showed a good linearity for AA measurement, as depicted in Fig. 3. The currents recorded clearly increased with increasing AA concentration in solution (Fig. 3(A)), and were linear with AA concentration within a range from 0.01 to $1.00 \mathrm{mM}(I / \mu \mathrm{A}=28.02 C / \mathrm{mM}-0.39, \gamma=0.9990)$ (Fig. 3(B)). The detection limit, based on a signal-to-noise ratio of 3, was calculated to be $5.0 \mu \mathrm{M}$. The activated electrode was also stable for repetitive use, and the sensitivity remained essentially identical after the daily measurements of AA standard $(0.10 \mathrm{mM})$ for 4-6 h on at least consecutive 10 days. On the other hand, the electrode showed good reproducibility for the determination of AA, which was illustrated by the small relative standard deviation (RSD) obtained with the same electrode used for the measurements of AA for several times. For example, the RSD recorded for the AA standard $(0.10 \mathrm{mM})$ was calculated to be $2.3 \%(n=10)$. The good performance of activated SPCEs mentioned above is indeed advantageous to be applied in determination of AA in real samples. 


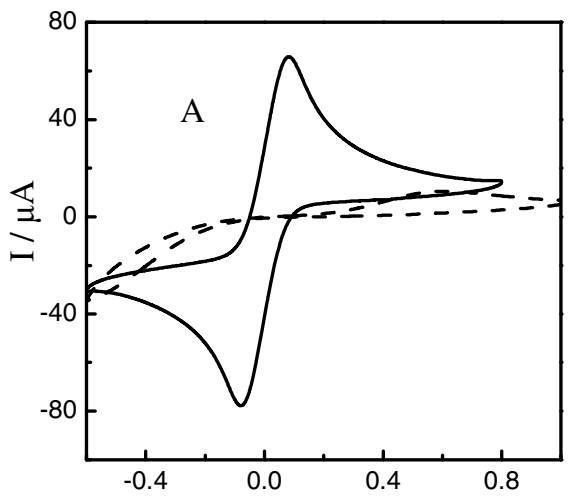

$\mathrm{E} / \mathrm{V}$ vs. $\mathrm{Ag} / \mathrm{AgCl}$

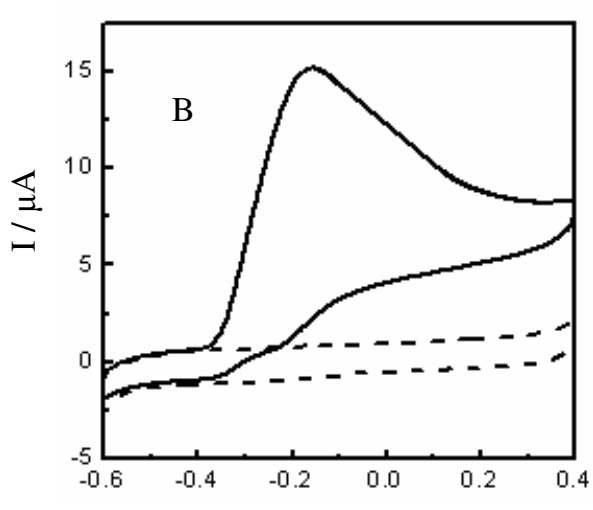

$\mathrm{E} / \mathrm{V}$ vs. $\mathrm{Ag} / \mathrm{AgCl}$

Fig. 2. (A) Typical cyclic voltammograms (CVs) at a fresh SPCE in $0.1 \mathrm{M}$ phosphate buffer ( $\mathrm{pH}$ 7.0) containing $5 \mathrm{mM} \mathrm{K} \mathrm{Fe}_{3}(\mathrm{CN})_{6}$ before (dashed line) and after (solid line) electrochemical pretreatment. Scan rate, $100 \mathrm{mV} \mathrm{s}^{-1}$. (B) CVs obtained at a fresh SPCE in $0.1 \mathrm{M}$ phosphate buffer $(\mathrm{pH} 7.0)$ in the absence (dashed line) and presence (solid line) of $0.50 \mathrm{mM}$ AA after electrochemical pretreatment. Scan rate, $100 \mathrm{mV} \mathrm{s}^{-1}$.

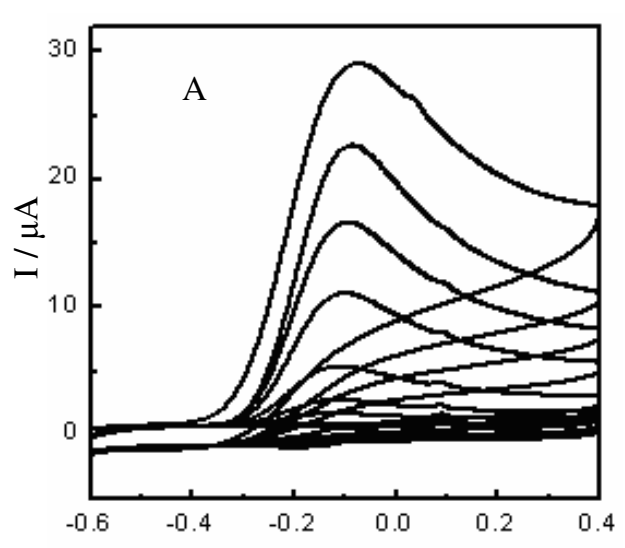

$\mathrm{E} / \mathrm{V}$ vs. $\mathrm{Ag} / \mathrm{AgCl}$

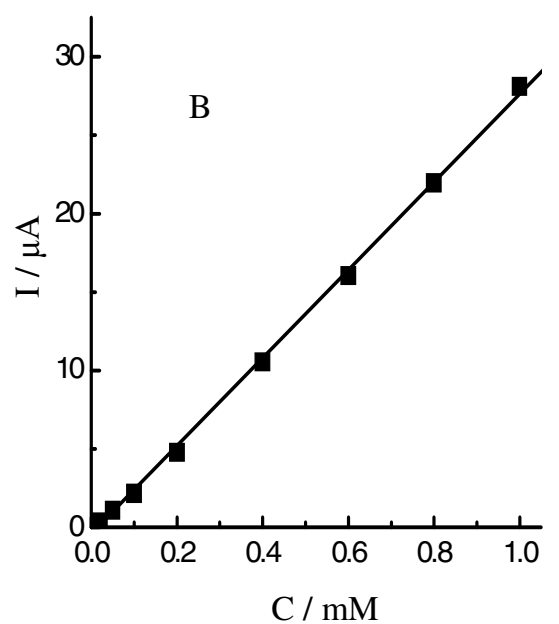

Fig. 3. (A) Typical CVs obtained at activated SPCE in $0.1 \mathrm{M}$ phosphate buffer (pH 7.0) containing AA with different concentrations of $0.00,0.01,0.02,0.05,0.10,0.20,0.40,0.60,0.80$ and $1.00 \mathrm{mM}$. Scan rate, $100 \mathrm{mV} \mathrm{s}^{-1}$. (B) Plot of current response vs the concentration of AA.

\subsection{Analysis of AA in Cabbage and Orange}

As detailedly described in experimental section, the AA content in cabbage and orange samples was analyzed by use of electrochemically pretreated SPCEs. As displayed in Fig. 4, the current response obtained for the addition of cabbage (Fig. 4(A)) and orange sample (Fig. 4(B)) showed well-defined shape of voltammograms, which 
were remarkably the same as the one obtained in AA standard solution before the real sample analysis. According to the oxidation current of real sample, AA content can be calculated in terms of the plot of sensor calibration in Fig. 3(B).

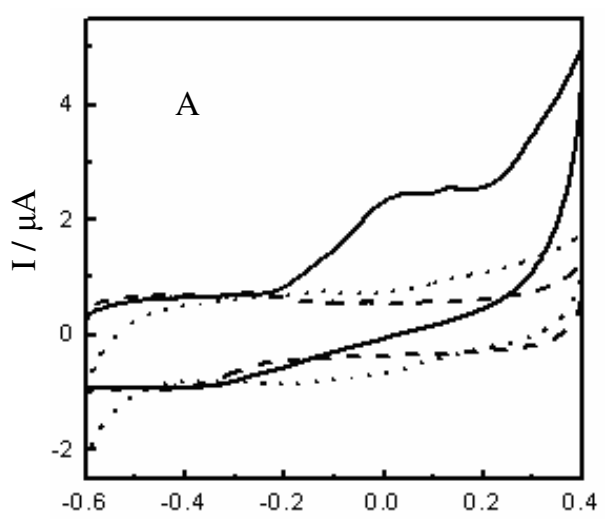

$\mathrm{E} / \mathrm{V}$ vs. $\mathrm{Ag} / \mathrm{AgCl}$

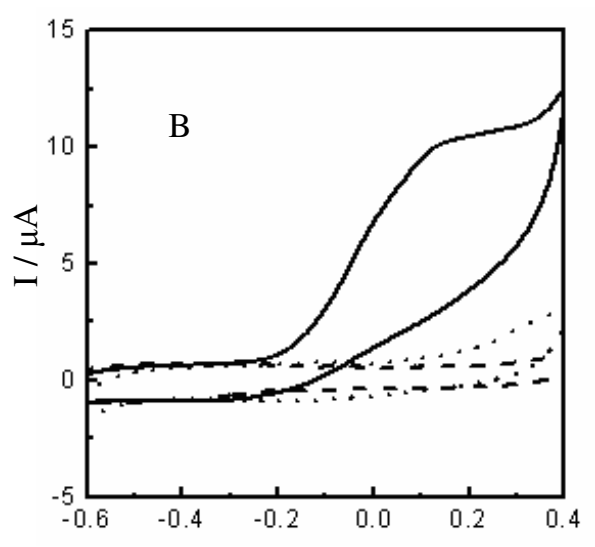

$\mathrm{E} / \mathrm{V}$ vs. $\mathrm{Ag} / \mathrm{AgCl}$

Fig. 4. CVs obtained at activated SPCEs in $0.1 \mathrm{M}$ phosphate buffer (pH 7.0) in the absence (dashed lines) and presence (solid lines) of diluted cabbage sample (A) and orange sample (B). The dotted lines in (A) and (B) refer to the responses of the mixture of AAox and each diluted sample after $15 \mathrm{~min}$, respectively. Scan rate, $100 \mathrm{mV} \mathrm{s}^{-1}$.

Considering the importance of the specificity of the AA determination in the real sample analysis demonstrated in this study, we further confirmed such a critical factor after the determination of AA content in both cabbage and orange by using AAOx to specifically catalyze the oxidation of AA [20]. The dotted lines in Fig. 4 showed the current response of a mixture of cabbage or orange with AAOx. As shown in Fig. 4(A) and Fig. 4(B), the presence of AAOx into the sample resulted in a clear decrease in the current response almost to the baseline level, confirming that it was feasible to selectively determine AA virtually interference-free from other electroactive species coexisting in the cabbage and orange.

In this study, the results obtained via an electrochemical voltammetric method have also been compared with those obtained using classical HPLC-UV analysis, in order

Table 1. Method comparison

\begin{tabular}{ccccc}
\hline \multirow{2}{*}{ sample } & \multicolumn{2}{c}{ cabbage $(\mathrm{mg} / \mathrm{kg})$} & \multicolumn{2}{c}{ orange $(\mathrm{mg} / \mathrm{kg})$} \\
\cline { 2 - 5 } 1 & voltammetry & HPLC & voltammetry & HPLC \\
2 & 96.3 & 97.6 & 420 & 431 \\
3 & 98.9 & 100 & 435 & 425 \\
4 & 99.7 & 97.8 & 428 & 435 \\
mean & 101 & 98.9 & 439 & 427 \\
\hline
\end{tabular}


to validate its reliability for rapid determination of AA in fresh vegetables and fruits. As displayed in Table 1, the comparison of the results obtained by the proposed electrochemical method to those of the reference method demonstrated an excellent correlation. These demonstrations suggest that the analytical system described in this study could be useful for rapid, facile, and reliable determination of AA in food analysis and other fields with low cost.

\section{Conclusions}

By taking advantage of facile electrochemical pretreatment and disposable screenprinted carbon electrodes, we have successfully developed a new electrochemical method for voltammetric measurement of AA with electrochemically activated electrodes. The integrated disposable screen printed carbon electrode, which consists of a carbon counter electrode, a silver pseudo-reference electrode and a carbon working electrode, is amenable to rapid fabrication and mass production, which simplifies the determination equipment and reduces the cost. In addition, this method demonstrated here just requires simple activation of electrodes to enhance the selectivity of AA determination, which avoids any artificial modification and reduces the determination time. Specificity assay and method comparison have demonstrated the electrodes possess a high selectivity and could thus be used for selective and reliable measurement of AA at lower applied potential. The method has also found to be sensitive, reproducible, and thus essentially finds some interesting applications in rapid food analysis and other fields.

Acknowledgments. This research was financially supported by the National High Technology Research and Development Program (2010AA10Z403, 2007AA10Z202), and Beijing Municipal Science and Technology Commission Program (Z09090501040901).

\section{References}

1. Rice, M.E.: Ascorbate Regulation and Its Neuroprotective Role in the Brain. Trends Neurosci. 23, 209-216 (2000)

2. Han, J.-S., Kozukue, N., Young, K.-S., Lee, K.-R., Friedman, M.: Distribution of Ascorbic Acid in Potato Tubers and in Homeprocessed and Commercial Potato Foods. J. Agric. Food Chem. 52, 6516-6521 (2004)

3. Dürüst, N., Sümengen, D., Dürüst, Y.: Ascorbic Acid and Element Contents of Food of Trabzon (Turkey). J. Agric. Food Chem. 45, 2085-2087 (1997)

4. Verdini, R.A., Lagier, C.M.: Voltammetric Iodometric Titration of Ascorbic Acid with Dead-Stop End Point Detection in Fresh Vegetables and Fruit Samples. J. Agric. Food Chem. 48, 2812-2817 (2000)

5. Terry, L.A., White, S.F., Tigwell, L.J.: The Application of Biosensors to Fresh Produce and the Wider Food Industry. J. Agric. Food Chem. 53, 1309-1316 (2005)

6. Zhang, M., Liu, K., Gong, K., Su, L., Chen, Y., Mao, L.: Continuous On-Line Monitoring of Extracellular Ascorbate Depletion in the Rat Striatum Induced by Global Ischemia with Carbon Nanotube-Modified Glassy Carbon Electrode Integrated into a Thin-Layer Radial Flow Cell. Anal. Chem. 77, 6234-6242 (2005) 
7. Malinauskas, A., Garjonyte, R., Mažeikien, R., Jureviciūte, I.: Electrochemical Response of Ascorbic Acid at Conducting and Electrogenerated Polymer Modified Electrodes for Electroanalytical Applications: a Review. Talanta 64, 121-129 (2004)

8. Nassef, H.M., Civit, L., Fragoso, A., O’Sullivan, C.K.: Amperometric Sensing of Ascorbic Acid Using a Disposable Sscreen-Printed Electrode Modified with Electrografted o-Aminophenol Film. Analyst 133, 1736-1741 (2008)

9. Mello, L.D., Kubota, L.T.: Biosensors as a Tool for the Antioxidant Status Evaluation. Talanta 72, 335-348 (2007)

10. O'Connell, P.J., Gormally, C., Pravda, M., Guilbault, G.G.: Development of an amperometric ascorbic acid (Vitamin C) sensor based on electropolymerised aniline for pharmaceutical and food analysis. Anal. Chim. Acta 431, 239-247 (2001)

11. Castilho, R.F., Souza, E.B.R., Alfaya, R.V.S., Alfaya, A.A.S.: Meldola's Blue Immobilized on a $\mathrm{SiO}_{2} / \mathrm{SnO}_{2} /$ Phosphate Xerogel, a New Sensor for Determination of Ascorbic Acid in Medicine and Commercial Fruit Juice. Electroanalysis 20, 157-162 (2008)

12. Nassef, H.M., Civit, L., Fragoso, A., O'Sullivan, C.K.: Amperometric Determination of Ascorbic Acid in Real Samples Using a Disposable Screen-Printed Electrode Modified with Electrografted $o$-Aminophenol Film. J. Agric. Food Chem. 56, 10452-10455 (2008)

13. Zhang, M., Liu, K., Xiang, L., Lin, Y., Su, L., Mao, L.: Carbon Nanotube-Modified Carbon Fiber Microelectrodes for In Vivo Voltammetric Measurement of Ascorbic Acid in Rat Brain. Anal. Chem. 79, 6559-6565 (2007)

14. Crevillénl, A.G., Pumera, M., Gonzálezl, M.C., Escarpa, A.: Carbon Nanotube Disposable Detectors in Microchip Capillary Electrophoresis for Water-Soluble Vitamin Determination: Analytical Possibilities in Pharmaceutical Quality Control. Electrophoresis 29, 2997 3004 (2008)

15. Cui, G., Yoo, J.H., Lee, J.S., Yoo, J., Uhm, J.H., Nam, H.: Effect of Pre-Treatment on the Surface and Electrochemical Properties of Screen-Printed Carbon Paste Electrodes. Analyst 126, 1399-1403 (2001)

16. Robards, K., Antolovich, M.: Methods for Assessing the Authenticity of Orange Juice: a Review. Analyst 120, 1-28 (1995)

17. McCreery, R.L.: Electroananlytical Chemistry. Dekker, New York (1991)

18. Guo, T., McCreery, R.L.: Surface Chemistry and Electron-Transfer Kinetics of HydrogenModified Glassy Carbon Electrodes. Anal. Chem. 71, 1553-1560 (1999)

19. Prasad, K.S., Muthuraman, G., Zen, J.-M.: The Role of Oxygen Functionalities and Edge Plane Sites on Screen-Printed Carbon Electrodes for Simultaneous Determination of Dopamine, Uric Acid and Ascorbic Acid. Electrochem. Commun. 10, 559-563 (2008)

20. Akyilmaz, E., Dinçkaya, E.: A New Enzyme Electrode Based on Ascorbate Oxidase Immobilized in Gelatin for Specific Determination of L-Ascorbic Acid. Talanta 50, 87-93 (1999) 